\title{
The controllability for the semi-discrete wave equation with a finite element method
}

Guojie Zheng ${ }^{1,2}$ and Xin $\mathrm{Yu}^{3 *}$

\author{
"Correspondence: yuxin@zju.edu.cn \\ ${ }^{3}$ Ningbo Institute of Technology, \\ Zhejiang University, Ningbo, \\ 315100, P.R. China \\ Full list of author information is \\ available at the end of the article
}

\begin{abstract}
In this paper, we study the controllability problem of the semi-discrete internally controlled one-dimensional wave equation with the finite element method. We derive the observability inequality and prove the exact controllability for the semi-discrete internally controlled wave equation, with the controls taken from a finite dimensional space.
\end{abstract}

MSC: 49K20; 35J65

Keywords: wave equation; finite element approximation; observability inequality

\section{Introduction}

In this paper, we discuss the topic related to the controllability for the semi-discrete internally controlled one-dimensional wave equations. First of all, we introduce certain notations. Let $\omega$ be an open and nonempty subset of $(0,1)$. Define an operator $E: L^{2}(\omega) \rightarrow$ $L^{2}(0,1)$ by

$$
E(f)(x)= \begin{cases}f(x) & \text { if } x \in \omega \\ 0 & \text { if } x \in(0,1) \backslash \omega\end{cases}
$$

for any $f \in L^{2}(\omega)$. Let $T>0$. The controlled wave equation, which we study in this paper, is as follows:

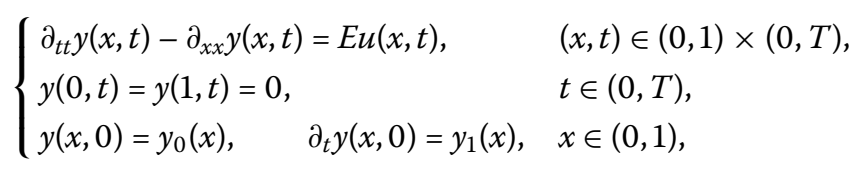

where the initial value $\left(y_{0}, y_{1}\right)$ belongs to $H_{0}^{1}(0,1) \times L^{2}(0,1)$ and $u(\cdot)$ is a control function taken from the space $L^{2}\left(0, T ; L^{2}(\omega)\right)$.

System (1.1) is said to be exactly controllable from the initial value $\left(y_{0}, y_{1}\right) \in H_{0}^{1}(0,1) \times$ $L^{2}(0,1)$ in time $T$ if there exists a control function $u(\cdot) \in L^{2}\left(0, T ; L^{2}(\omega)\right)$ such that the solution of $(1.1)$ matches that $\left(y(T), \partial_{t} y(T)\right)=(0,0)$. We have already known that the controllability property for the above continuous one-dimensional wave equation holds for any given $T>2$ (see [1]).

In this work, we mainly focus on the issue of the controllability property of (1.1) under numerical approximation schemes with the finite element method. To this end, now we introduce the basis functions of the finite element space. Let $h$ be a small enough positive 
number. Corresponding to each given $h$, we take nodal points $x_{i}$, with $i=0,1, \ldots, N_{h}$, over the interval $[0,1]$ such that

$$
0=x_{0}<x_{1}<\cdots<x_{N_{h}-1}<x_{N_{h}}=1
$$

and

$$
h=\max _{1 \leq j \leq N_{h}} h_{j}, \quad \text { where } h_{j}=\left(x_{j}-x_{j-1}\right) .
$$

Let

$$
\omega=\left(x_{k}, x_{k+p}\right) \quad \text { for some } k \text { and } p \text { with } k \geq 1, p \geq 1 \text { and }(k+p)<N_{h} \text {. }
$$

Then we can define the basis function $\phi_{j}$ by

$$
\phi_{j}(x)= \begin{cases}\frac{x-x_{j-1}}{h_{j}}, & x \in\left[x_{j-1}, x_{j}\right], \\ \frac{x_{j+1}-x}{h_{j+1}}, & x \in\left[x_{j}, x_{j+1}\right], \\ 0, & x \in[0,1] \backslash\left[x_{j-1}, x_{j+1}\right] .\end{cases}
$$

Corresponding to the state space $L^{2}(0,1)$, we build the finite element space as

$$
V_{0}^{h}=\operatorname{span}\left\{\phi_{1}, \phi_{2}, \ldots, \phi_{N_{h}-1}\right\} .
$$

Obviously, it is a subspace of $H_{0}^{1}(0,1)$. Let $P_{h}$ be the $L^{2}$-projection from $L^{2}(0,1)$ to $V_{0}^{h}$, namely

$$
\left\langle P_{h} v, v_{h}\right\rangle=\left\langle v, v_{h}\right\rangle, \quad \forall v \in L^{2}(0,1), v_{h} \in V_{0}^{h} .
$$

Corresponding to the control space $L^{2}(\omega)$, we define the finite element space by

$$
\tilde{V}^{h}=\left\{w_{h} ; w_{h}=\chi_{\omega} v_{h} \text { for some } v_{h} \in V_{0}^{h}\right\}
$$

Throughout this paper, $\chi_{\omega}$ will be treated as an operator from $L^{2}(0,1)$ to $L^{2}(\omega)$, by setting $\chi_{\omega}(f)=\left.f\right|_{\omega}$ for all $f \in L^{2}(0,1)$. Clearly, $\widetilde{V}^{h}$ is a subspace of $H^{1}(\omega)$.

Write $E_{h}$ for the restriction of the operator $E$ over $\widetilde{V}^{h}$, and project equation (1.1) into the following controlled ordinary differential equations:

$$
\left\{\begin{array}{l}
y_{h}^{\prime \prime}(t)-\triangle_{h} y_{h}(t)=P_{h} \circ E_{h}\left(u_{h}(t)\right), \quad t>0, \\
y_{h}(0)=y_{0}^{h}, \quad y_{h}^{\prime}(0)=y_{1}^{h} .
\end{array}\right.
$$

Here, the initial value $\left(y_{0}^{h}, y_{1}^{h}\right)$ belongs to $V_{0}^{h} \times V_{0}^{h}$, the control $u_{h}(\cdot)$ is taken from the space $L^{2}\left(0,+\infty ; \widetilde{V}^{h}\right)$, and the operator $-\triangle_{h}: V_{0}^{h} \rightarrow V_{0}^{h}$ is defined by

$$
\left\langle-\Delta_{h} v_{h}, w_{h}\right\rangle=\int_{\Omega} \nabla v_{h} \cdot \nabla w_{h} d x \quad \text { for any } v_{h}, w_{h} \in V_{0}^{h}
$$

In this paper, we mainly deal with the controllability for semi-discrete system (1.4). The main result of the paper is presented as follows. 
Theorem 1.1 For each $T>0$, controlled system (1.4) is exactly controllable in time $T$. Namely, there exists a control function $v_{h}(\cdot) \in L^{2}\left(0,+\infty ; \widetilde{V}^{h}\right)$ such that the solution of (1.4) satisfies $\left(y_{h}(T), y_{h}^{\prime}(T)\right)=(0,0)$.

Remark 1.2 In this paper, our main purpose is to discuss whether or not the semi-discrete internally controlled systems have the exactly controllable property which the original controlled systems have. This is a very valuable problem in control theory. In [2], the authors established such a controllability result for the semi-discrete one-dimensional boundary controlled wave equation by the numerical approximation method. The authors also got that the semi-discrete systems are not uniformly controllable as the discretization parameter $h$ goes to zero. The main differences between [2] and our paper are as follows. In [2], the authors focused on a one-dimensional boundary controlled wave equation and they obtained the controllability for the semi-discrete wave system, with the controls taken from an infinite dimensional space. In our paper, we discuss an internally controlled onedimensional wave equation. In this case, we obtain the exact controllability of the semidiscrete wave equation, with the controls taken from a finite dimensional space. Regarding other works related to this problem, we would like to mention [3, 4], and [5].

The rest of the paper is structured as follows. In Section 2, we give a sufficient condition for controllability. By making use of this sufficient condition presented in Section 2, we provide the proof of Theorem 1.1 in Section 3.

\section{The controllability and observability property}

We first introduce the following auxiliary system.

$$
\left\{\begin{array}{l}
y_{h}^{\prime \prime}(t)-\triangle_{h} y_{h}(t)=P_{h} \circ E(u(t)), \quad t>0 \\
y_{h}(0)=y_{0}^{h}, \quad y_{h}^{\prime}(0)=y_{1}^{h}
\end{array}\right.
$$

where the initial data $\left(y_{0}^{h}, y_{1}^{h}\right) \in V_{0}^{h} \times V_{0}^{h}$. Clearly, it is a controlled system governed by ordinary differential equations. However, the control functions for this system are taken from the infinite dimensional space $L^{2}\left(0, T ; L^{2}(\omega)\right)$.

In this section, we discuss some controllability result for system (2.1). More concisely, a sufficient condition for the exact controllability property of (2.1) will be presented. The proofs of the following Lemmas 2.1, 2.2 and 2.4 can be found in [6].

For any $\left(\varphi_{0}^{h}, \varphi_{1}^{h}\right) \in V_{0}^{h} \times V_{0}^{h}$, consider the following homogeneous equation:

$$
\left\{\begin{array}{l}
\varphi_{h}^{\prime \prime}(t)-\triangle_{h} \varphi_{h}(t)=0, \quad t>0, \\
\varphi_{h}(0)=\varphi_{0}^{h}, \quad \varphi_{h}^{\prime}(0)=\varphi_{1}^{h} .
\end{array}\right.
$$

Lemma 2.1 The control $u \in L^{2}\left(0, T ; L^{2}(\omega)\right)$ drives the initial data $\left(y_{0}^{h}, y_{1}^{h}\right)$ of controlled system (2.1) to zero in time $T$ if and only if

$$
\int_{0}^{T} \int_{\omega} \varphi_{h} u d x d t=\int_{0}^{1} \varphi_{1}^{h} y_{h}(0) d x-\int_{0}^{1} \varphi_{0}^{h} y_{h}^{\prime}(0) d x
$$

for all $\left(\varphi_{0}^{h}, \varphi_{1}^{h}\right) \in V_{0}^{h} \times V_{0}^{h}$, where $\varphi_{h}$ is the corresponding solution of equation (2.2). 
Next, we define a functional $\mathcal{J}$ from $V_{0}^{h} \times V_{0}^{h}$ to $\mathbb{R}$ by setting

$$
\mathcal{J}\left(\varphi_{0}^{h}, \varphi_{1}^{h}\right)=\frac{1}{2} \int_{0}^{T} \int_{\omega}\left|\varphi_{h}\right|^{2} d x d t+\int_{0}^{1} \varphi_{0}^{h} y_{h}^{\prime}(0) d x-\int_{0}^{1} \varphi_{1}^{h}(0) y_{h}(0) d x
$$

where $\varphi_{h}$ is the solution of (2.2) with initial data $\left(\varphi_{0}^{h}, \varphi_{1}^{h}\right) \in V_{0}^{h} \times V_{0}^{h}$.

We have the following result.

Lemma 2.2 Suppose that $\left(\widehat{\varphi}_{0}^{h}, \widehat{\varphi}_{1}^{h}\right) \in V_{0}^{h} \times V_{0}^{h}$ is a minimizer of $\mathcal{J}$. If $\widehat{\varphi}_{h}$ is the corresponding solution of equation (2.2) with initial $\left(\widehat{\varphi}_{0}^{h}, \widehat{\varphi}_{1}^{h}\right)$, then

$$
u=\chi_{\omega} \widehat{\varphi}_{h}
$$

is a control which drives the initial data $\left(y_{0}^{h}, y_{1}^{h}\right)$ of controlled system (2.1) to zero in time T.

To get the sufficient condition that ensures the existence of a minimizer for $\mathcal{J}$, we need to give the following definition.

Definition 2.3 Equation (2.2) is observable in time $T$ if there exists a positive constant $L$ such that the following inequality holds:

$$
L\left\|\left(\varphi_{0}^{h}, \varphi_{1}^{h}\right)\right\|_{H_{0}^{1}(0,1) \times H^{-1}(0,1)}^{2} \leq \int_{0}^{T} \int_{\omega}\left|\varphi_{h}\right|^{2} d x d t
$$

for any $\left(\varphi_{0}^{h}, \varphi_{1}^{h}\right) \in V_{0}^{h} \times V_{0}^{h}$, where $\varphi_{h}$ is the solution of (2.2) with initial data $\left(\varphi_{0}^{h}, \varphi_{1}^{h}\right)$.

Inequality (2.6) is called observability inequality. The following conclusion shows that observability inequality (2.6) is the sufficient condition for the exact controllability of system (2.1).

Lemma 2.4 Suppose equation (2.2) is observable in time $T$. Then the functional $\mathcal{J}$ defined by (2.4) has a unique minimizer $\left(\widehat{\varphi}_{0}^{h}, \widehat{\varphi}_{1}^{h}\right) \in V_{0}^{h} \times V_{0}^{h}$.

\section{The proof of Theorem 1.1}

Before giving the proof of the main result, we first present some preliminary lemmas. Assume that all distinct eigenvalues of the operator $-\triangle_{h}$ are $\lambda_{1}^{h}, \lambda_{2}^{h}, \ldots, \lambda_{q}^{h}, 0<\lambda_{1}^{h}<\lambda_{2}^{h}<$ $\cdots<\lambda_{q}^{h}$. For any given eigenvalue $\lambda_{s}^{h}, s \in\{1,2, \ldots, q\}$, let $l_{s}$ be its multiplicity and $W_{s}^{h}$ be its eigenspace, with an orthogonal basis $\left\{e_{s, 1}^{h}, e_{s, 2}^{h}, \ldots, e_{s, l_{s}}^{h}\right\}$. It is easy to see that the family

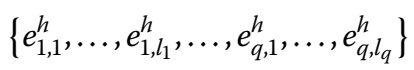

forms an orthogonal basis of $V_{0}^{h}$. The following two results are quoted from [7]. They will be used later.

Lemma 3.1 For any non-zero vector $\xi_{h}$ in the space $V_{0}^{h}$, we have $\xi_{h}=\sum_{s=1}^{q} r_{s} f_{s}^{h}$, where $f_{s}^{h}$, $s \in\{1,2, \ldots, q\}$, is a normalized eigenfunction in the eigenspace $W_{s}^{h}$, and $r_{1}, r_{2}, \ldots, r_{q}$ are real numbers satisfying $\sum_{s=1}^{q} r_{s}^{2}=\left\|\xi_{h}\right\|^{2}$, where $\|\cdot\|$ denotes the usual norm of $L^{2}(0,1)$. 
Theorem 3.2 Suppose that $X^{h}$ is an eigenfunction to the operator $-\triangle_{h}$, and $\omega$ is an open and nonempty subset of $(0,1)$. Then $\chi_{\omega} X^{h} \neq 0$.

Now, we will prove the controllability for system (2.1).

Theorem 3.3 For each $T>0$, the solution of semi-discrete system (2.2) satisfies the following inequality:

$$
L\left\|\left(\varphi_{0}^{h}, \varphi_{1}^{h}\right)\right\|_{L^{2}(0,1) \times L^{2}(0,1)}^{2} \leq \int_{0}^{T} \int_{\omega}\left|\varphi_{h}\right|^{2} d x d t .
$$

Remark 3.4 Since $V_{0}^{h} \times V_{0}^{h}$ is a finite dimensional space, thus all norms of this space are equivalent, and then we can get that inequality (3.1) implies observability of semi-discrete system (2.2).

Proof For any given $T>0$, consider the following function $F: V_{0}^{h} \times V_{0}^{h} \rightarrow \mathbb{R}$ defined by

$$
F\left(\varphi_{0}^{h}, \varphi_{1}^{h}\right)=\int_{0}^{T} \int_{\omega}\left|\varphi_{h}\right|^{2} d x d t
$$

where $\varphi_{h}$ is the solution of (2.2) with initial data $\left(\varphi_{0}^{h}, \varphi_{1}^{h}\right)$. Clearly, $F$ is continuous. To prove inequality (3.1), it suffices to show that we can find a positive constant $L(h, T)$, where $L(h, T)$ only depends on $h$ and $T$, such that

$$
\min \left\{F\left(\varphi_{0}^{h}, \varphi_{1}^{h}\right) ;\left\|\left(\varphi_{0}^{h}, \varphi_{1}^{h}\right)\right\|_{L^{2}(0,1) \times L^{2}(0,1)}=1\right\} \geq L(h, T) .
$$

To this end, we will give a proof by contradiction. Suppose that there is a unit vector $\left(w_{0}^{h}, w_{1}^{h}\right)$ in $V_{0}^{h} \times V_{0}^{h}$ such that $F\left(w_{0}^{h}, w_{1}^{h}\right)=0$. Since $\left\|\left(w_{0}^{h}, w_{1}^{h}\right)\right\|_{L^{2}(0,1) \times L^{2}(0,1)}=1$, at least one of $w_{0}^{h}$ and $w_{1}^{h}$ is not 0 . Without loss of generality, we can assume that $w_{0}^{h} \neq 0$. According to Lemma $3.1, w_{0}^{h}$ can be presented by

$$
w_{0}^{h}=\sum_{s=1}^{q} \xi_{s} f_{s}^{h},
$$

where $f_{s}^{h}$, with $s \in\{1,2, \ldots, q\}$, is a normalized eigenfunction in the eigenspace $W_{s}^{h}$, and $\xi_{1}, \xi_{2}, \ldots, \xi_{q}$ are real numbers satisfying

$$
\sum_{s=1}^{q} \xi_{s}^{2}=\left\|w_{0}^{h}\right\|_{L^{2}(0,1)}^{2} \neq 0
$$

Now, noting that $V_{h}^{0}$ is an $N_{h}-1$ dimensional space, we can choose $f_{q+1}^{h}, \ldots, f_{N_{h}-1}^{h}$ which are normalized eigenfunctions of $-\triangle_{h}$ such that $f_{1}^{h}, \ldots, f_{q}^{h}, f_{q+1}^{h}, \ldots, f_{N_{h}-1}^{h}$ constitute a complete standard orthogonal basis of $V_{h}^{0}$. Let $\lambda_{1}^{h}, \ldots, \lambda_{N_{h}-1}^{h}$ be $N_{h}-1$ corresponding eigenvalues to eigenfunctions $f_{1}^{h}, \ldots, f_{q}^{h}, f_{q+1}^{h}, \ldots, f_{N_{h}-1}^{h}$.

Now, for $\left(w_{0}^{h}, w_{1}^{h}\right) \in V_{0}^{h} \times V_{0}^{h}$, we can write

$$
w_{1}^{h}=\sum_{s=1}^{N_{h}-1} \eta_{s} f_{s}^{h}
$$


and we can rewrite

$$
w_{0}^{h}=\sum_{s=1}^{N_{h}-1} \xi_{s} f_{s}^{h},
$$

where $\xi_{q+1}=\cdots=\xi_{N_{h}-1}=0$. By the classical theory of ODEs, equation (2.2) has a unique solution

$$
\varphi_{h}(t)=\sum_{s=1}^{N_{h}-1}\left\{\xi_{s} \cos \left(\sqrt{\lambda_{s}^{h}} t\right)+\frac{\eta_{s}}{\sqrt{\lambda_{s}^{h}}} \sin \left(\sqrt{\lambda_{s}^{h}} t\right)\right\} f_{s}^{h} .
$$

This, together with the definition of the function $F$ and the assumption that $F\left(w_{0}^{h}, w_{1}^{h}\right)=0$, indicates that

$$
\begin{aligned}
0 & =F\left(w_{0}^{h}, w_{1}^{h}\right) \\
& =\int_{0}^{T} \int_{\omega}\left|\varphi_{h}\right|^{2} d x d t \\
& =\int_{0}^{T}\left\|\sum_{s=1}^{N_{h}-1}\left\{\xi_{s} \cos \left(\sqrt{\lambda_{s}^{h}} t\right)+\frac{\eta_{s}}{\sqrt{\lambda_{s}^{h}}} \sin \left(\sqrt{\lambda_{s}^{h}} t\right)\right\} \chi_{\omega} f_{s}^{h}\right\|_{L^{2}(\omega)}^{2} d t .
\end{aligned}
$$

Hence, we necessarily have that

$$
\sum_{s=1}^{N_{h}-1}\left\{\xi_{s} \cos \left(\sqrt{\lambda_{s}^{h}} t\right)+\frac{\eta_{s}}{\sqrt{\lambda_{s}^{h}}} \sin \left(\sqrt{\lambda_{s}^{h}} t\right)\right\} \chi_{\omega} f_{s}^{h}=0 \quad \text { in } L^{2}(\omega) \text { for all } t \in[0, T]
$$

In the following, we are going to prove that

$$
\xi_{s}=0 \quad \text { for each } s \in\{1,2, \ldots, q\}
$$

which leads to a contradiction to the assumption that $w_{0}^{h} \neq 0$, and then we can complete the proof of (3.2).

By taking $t=0$ in (3.4) and noting $\varphi_{h}(0)=w_{0}^{h}$, we can get

$$
\sum_{s=1}^{N_{h}-1} \xi_{s} \chi_{\omega} f_{s}^{h}=\sum_{s=1}^{q} \xi_{s} \chi_{\omega} f_{s}^{h}=0 \quad \text { in } L^{2}(\omega),
$$

where we use the fact that $\xi_{q+1}=\cdots=\xi_{N_{h}-1}=0$. Calculating the derivations twice to (3.4) and taking $t=0$, we can get

$$
\sum_{s=1}^{N_{h}-1} \lambda_{s}^{h} \xi_{s} \chi_{\omega} f_{s}^{h}=\sum_{s=1}^{q} \lambda_{s}^{h} \xi_{s} \chi_{\omega} f_{s}^{h}=0 \quad \text { in } L^{2}(\omega) .
$$

Thus, by induction, we can get that

$$
\sum_{s=1}^{q}\left(\lambda_{s}^{h}\right)^{r} \xi_{s} \chi_{\omega} f_{s}^{h}=0 \quad \text { in } L^{2}(\omega)
$$

where $r=0,1,2, \ldots$. 
Noting that $f_{s}^{h} \neq 0, s=1,2, \ldots, q$, are the eigenfunctions of $-\triangle_{h}$, we can see from Theorem 3.2 that $\chi_{\omega} f_{s}^{h} \neq 0$ for $s=1,2, \ldots, q$. Therefore, we can assume, without loss of generality, that $\chi_{\omega} f_{1}^{h}, \ldots, \chi_{\omega} f_{\alpha}^{h}$ with $1 \leq \alpha \leq q$ are linear independent in $L^{2}(\omega)$, and

$$
\operatorname{span}\left\{\chi_{\omega} f_{1}^{h}, \ldots, \chi_{\omega} f_{\alpha}^{h}\right\}=\operatorname{span}\left\{\chi_{\omega} f_{1}^{h}, \ldots, \chi_{\omega} f_{q}^{h}\right\}
$$

With regard to the number $\alpha$, there are only two possibilities: it either is equal to $q$ or is less than $q$. If $\alpha=q$, (3.5) follows immediately from (3.6). If $\alpha<q$, we have the following presentation:

$$
\chi_{\omega} f_{j}^{h}=\sum_{s=1}^{\alpha} a_{j s} \chi_{\omega} f_{s}^{h}, \quad j=\alpha+1, \ldots, q
$$

where $a_{j s}, s=1,2, \ldots, \alpha, j=\alpha+1, \ldots, q$, are real numbers. Since $\chi_{\omega} f_{j}^{h} \neq 0$ for all $j=\alpha+$ $1, \ldots, q$, we derive, from (3.7), the following fact:

For each $j \in\{\alpha+1, \ldots, q\}$, there is a number $s(j) \in\{1, \ldots, \alpha\}$ such that $a_{j s(j)} \neq 0$.

On the other hand, combining (3.6) with (3.7) leads to

$$
\begin{aligned}
0 & =\sum_{s=1}^{q}\left(\lambda_{s}^{h}\right)^{r} \xi_{s} \chi_{\omega} f_{s}^{h} \\
& =\sum_{s=1}^{\alpha}\left(\lambda_{s}^{h}\right)^{r} \xi_{s} \chi_{\omega} f_{s}^{h}+\sum_{j=\alpha+1}^{q}\left(\lambda_{j}^{h}\right)^{r} \xi_{j} \chi_{\omega} f_{j}^{h} \\
& =\sum_{s=1}^{\alpha}\left(\left(\lambda_{s}^{h}\right)^{r} \xi_{s}+\sum_{j=\alpha+1}^{q}\left(\lambda_{j}^{h}\right)^{r} \xi_{j} a_{j s}\right) \chi_{\omega} f_{s}^{h} \quad \text { in } L^{2}(\omega) .
\end{aligned}
$$

Since $\chi_{\omega} f_{1}^{h}, \ldots, \chi_{\omega} f_{\alpha}^{h}$ are linear independent in $L^{2}(\omega)$, it follows from the above identity that

$$
0=\left(\lambda_{s}^{h}\right)^{r} \xi_{s}+\sum_{j=\alpha+1}^{q}\left(\lambda_{j}^{h}\right)^{r} \xi_{j} a_{j s} \quad \text { for all } s=1, \ldots, \alpha, r=0,1,2, \ldots
$$

Because $\lambda_{1}^{h}, \ldots, \lambda_{q}^{h}$ are distinct positive numbers, we can deduce immediately from (3.9) that

$$
\xi_{s}=0 \quad \text { for all } s=1, \ldots, \alpha
$$

and that

$$
\xi_{j} a_{j s}=0 \quad \text { for all } s=1, \ldots, \alpha, \text { and all } j=\alpha+1, \ldots, q \text {. }
$$

In the above second identity, corresponding to each $j \in\{\alpha+1, \ldots, q\}$, we can take $s$ as the number $s(j)$ given in (3.8). Then it follows that $\xi_{j}=0$ for all $j \in\{\alpha+1, \ldots, q\}$. Hence, we prove (3.5) and finish the proof for this theorem. 
Proof of Theorem 1.1 According to Lemma 2.2, Lemma 2.4, and Theorem 3.3, we have that system (2.1) is controllable in time $T$. Suppose that $\left(\widehat{\varphi}_{0}^{h}, \widehat{\varphi}_{1}^{h}\right) \in V_{0}^{h} \times V_{0}^{h}$ is a minimizer of $\mathcal{J}$. If $\widehat{\varphi}_{h}$ is the corresponding solution of equation (2.2) with initial data $\left(\widehat{\varphi}_{0}^{h}, \widehat{\varphi}_{1}^{h}\right)$, then

$$
u=\chi_{\omega} \widehat{\varphi}_{h}
$$

is a control which drives the initial data $\left(y_{0}^{h}, y_{1}^{h}\right)$ of controlled system (2.1) to zero in time $T$. It is easy to see that $\chi_{\omega} \widehat{\varphi}_{h} \in L^{2}\left(0,+\infty ; \widetilde{V}^{h}\right)$. This completes the proof of this theorem.

Competing interests

The authors declare that they have no competing interests.

\section{Authors' contributions}

GZ provided the questions. GZ and XY gave the proof for the main result together. All authors read and approved the final manuscript.

\section{Author details}

${ }^{1}$ College of Mathematics and Information Science, Henan Normal University, Xinxiang, 453007, P.R. China. ${ }^{2}$ School of Computational and Applied Mathematics, University of the Witwatersrand, Johannesburg, South Africa. ${ }^{3}$ Ningbo Institute of Technology, Zhejiang University, Ningbo, 315100, P.R. China.

\section{Acknowledgements}

The authors would like to thank professor Gengsheng Wang for his valuable suggestions on this paper. This work was partially supported by the National Natural Science Foundation of China (U1204105, 61203293), the Natural Science Foundation of Zhejiang (Y6110751), the Natural Science Foundation of Ningbo (2010A610096), the Key Foundation of Henan Educational Committee (13A120524, 12B120006), and the Fundamental and Frontier Technology Research Projects of Henan Province (132300410285).

\section{Received: 22 February 2013 Accepted: 22 May 2013 Published: 7 June 2013}

\section{References}

1. Bardos, C, Lebeau, G, Rauch, J: Sharp sufficient conditions for the observation, control and stabilization of waves from the boundary. SIAM J. Control Optim. 30, 1024-1065 (1992)

2. Infante, JA, Zuazua, E: Boundary observability for the space semi-discretization of the 1-D wave equation. ESAIM Math. Model. Numer. Anal. 33, 407-438 (1999)

3. Castro, C, Micu, S: Boundary controllability of a linear semi-discrete 1-D wave equation derived from a mixed finite element method. Numer. Math. 102, 413-462 (2006)

4. Castro, C, Micu, S: Numerical approximation of the boundary control for the wave equation with mixed finite elements in a square. IMA J. Numer. Anal. 28, 186-214 (2008)

5. Zuazua, E: Propagation, observation, control and numerical approximation of waves by finite difference method. SIAM Rev. 47, 197-243 (2005)

6. Micu, S, Zuazua, E: An introduction to the controllability of partial differential equations. In: Sari, T (ed.) Collection Travaux en Cours Hermannin Quelques Questions de Théorie du Contrôle, pp. 69-157 (2005)

7. Wang, G, Zheng, G: An approach to the optimal time for a time optimal control problem of an internally controlled heat equation. SIAM J. Control Optim. 50, 601-628 (2012) 